\title{
Maxillary Sinus Augmentation Combining Bio-Oss with the Bone Marrow Aspirate Concentrate: A Histomorphometric Study in Humans
}

\author{
Paulo José Pasquali, ${ }^{1}$ Marcelo Lucchesi Teixeira, ${ }^{2}$ \\ Thiago Altro de Oliveira, ${ }^{1}$ Luis Guilherme Scavone de Macedo, ${ }^{1}$ \\ Antonio Carlos Aloise, ${ }^{1}$ and André Antonio Pelegrine ${ }^{1}$ \\ ${ }^{1}$ Department of Implant Dentistry, São Leopoldo Mandic Dental School, 13 José Rocha Junqueira Street, 13045-755 Campinas, Brazil \\ ${ }^{2}$ Department of Prosthodontics, São Leopoldo Mandic Dental School, 13 José Rocha Junqueira Street, 13045-755 Campinas, Brazil
}

Correspondence should be addressed to André Antonio Pelegrine; pelegrineandre@gmail.com

Received 14 June 2015; Revised 3 September 2015; Accepted 27 September 2015

Academic Editor: Sean Peel

Copyright (C) 2015 Paulo José Pasquali et al. This is an open access article distributed under the Creative Commons Attribution License, which permits unrestricted use, distribution, and reproduction in any medium, provided the original work is properly cited.

Purpose. To investigate the regenerative results obtained with the association of bone marrow aspirate concentrate using the Bone Marrow Aspirate Concentrate (BMAC) method to a xenogeneic bone graft (Bio-Oss) in sinus floor elevation. Materials and Methods. Using a randomized controlled study design in eight consecutive patients (age of $55.4 \pm 9.2$ years), 16 sinus floor lift procedures were performed with Bio-Oss alone (control group, CG, $n=8$ ) or combined with bone marrow aspirate concentrate obtained via the BMAC method (test group, TG, $n=8$ ). Six months after the grafting procedures, bone biopsies were harvested during implant placement and were analyzed by histomorphometry. Results. Histomorphometric analysis revealed a significantly higher amount $(p<0.05)$ of vital mineralized tissue in TG when compared to the CG $(55.15 \pm 20.91 \%$ and $27.30 \pm 5.55 \%$, resp.). For nonvital mineralized tissue, TG presented a statistically higher level of Bio-Oss resorption $(p<0.05)$ when compared with the CG $(6.32 \pm 12.03 \%$ and $22.79 \pm 9.60 \%$, resp.). Both groups (TG and CG) showed no significantly different levels $(p>0.05)$ of nonmineralized tissue ( $38.53 \pm 13.08 \%$ and $49.90 \pm 7.64 \%$, resp.). Conclusion. The use of bone marrow concentrate obtained by BMAC method increased bone formation in sinus lift procedures.

\section{Introduction}

Inadequate bone quantity in the posterior maxilla secondary to pneumatization of the maxillary sinus and/or postextraction alveolar ridge resorption can compromise dental implant placement, therefore requiring site grafting prior to implant placement using techniques such as maxillary sinus floor elevation. The ideal material for bone grafting should be biocompatible, induce no host rejection, present no risk of disease transmission, promote support for bone regeneration, and have mechanical stability from the outset, which should be maintained throughout the healing period [1].

The maxillary sinus floor elevation technique described by Tatum and published by Boyne and James in 1980 [2] described autologous bone as the filling material for the sinus cavity, which is still regarded as the gold standard in bone reconstruction. If, on the one hand, autologous grafts present osteoconductive, osteoinductive, and osteogenic potential [3], on the other, they present some risks [4]. This has translated into a constant search for the development of biomaterials to substitute autologous bone grafts, for instance, xenografts $[5,6]$, homologous grafts $[7,8]$, and synthetic grafts $[9,10]$.

Bone substitute biomaterials lack cellularity, which has encouraged much research into the field of tissue engineering in order to combine autologous osteogenic cells with osteoconductive materials. Consequently, the bone marrow is currently the most explored source of autologous cells, [11] since it contains a number of bone regenerating cells, such as undifferentiated mesenchymal cells that can differentiate 


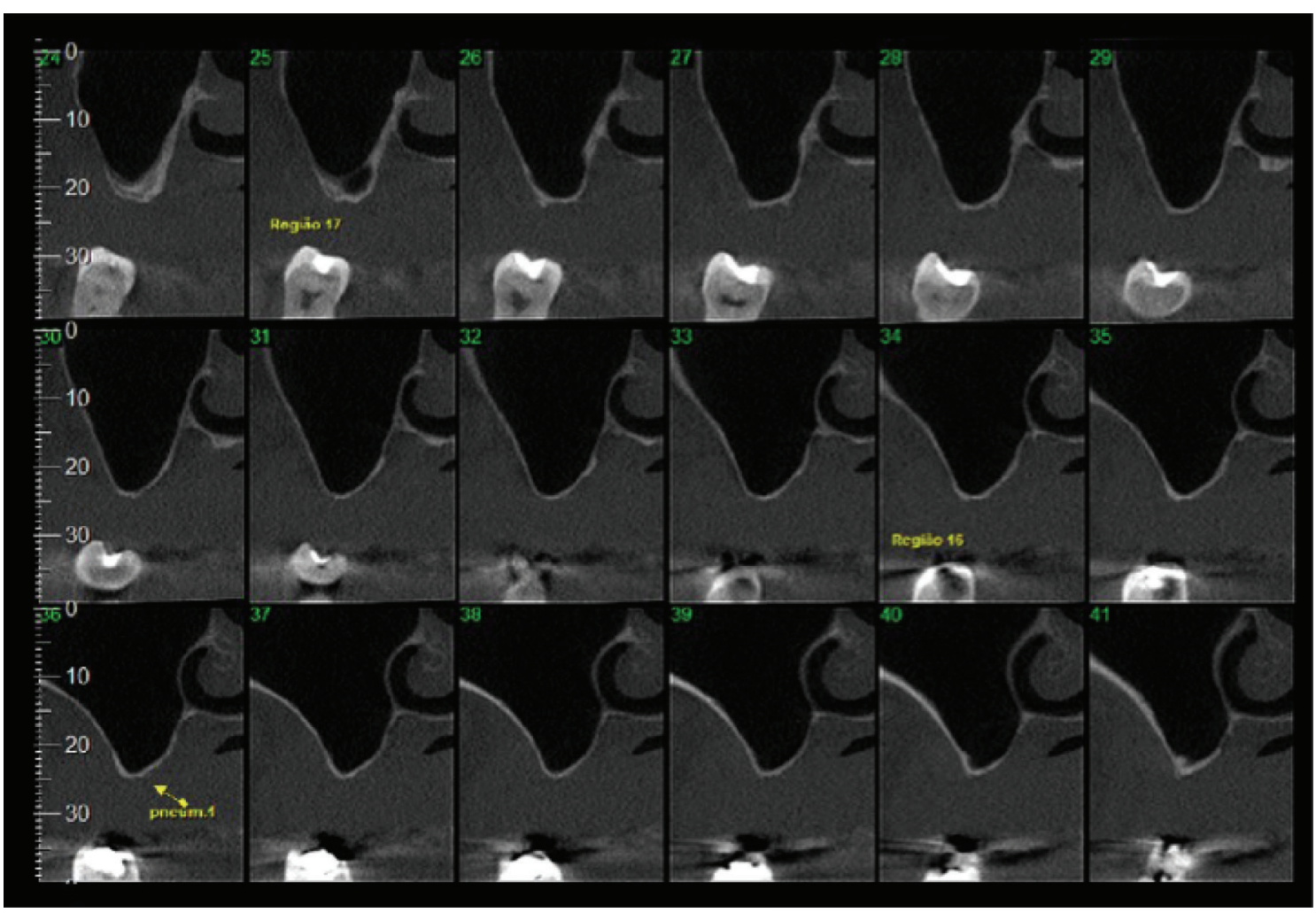

FIGURE 1: Cone-beam computed tomography scans of the posterior maxillary region.

into osteoblasts $[12,13]$. They also present an angiogenic potential due to the production and release of vascular endothelium growth factor [14], which is highly desirable for graft integration [15].

Osteoconductive graft enrichment in reconstructive surgery for maxillary sinus floor elevation can be performed using cells from a bone marrow aspirate concentrate obtained by centrifugation [16-19]. This method is regarded as simple and safe because it is performed using autologous material immediately before surgery [20].

Therefore, the aim of this study was to histomorphometrically evaluate the use of a bone xenograft enriched with autologous bone marrow aspirate concentrate (BMAC) for maxillary sinus floor lifting.

\section{Materials and Methods}

This study was conducted in the outpatient clinic of the Department of Implant Dentistry of the São Leopoldo Mandic Dental School (Campinas, SP, Brazil), upon approval by the research ethics committee $(694.065 / 2014)$ and free and informed consent forms for all the patients.

The inclusion criteria involved completely edentulous patients needing implants in the posterior maxillary region with no more than $4 \mathrm{~mm}$ of remaining alveolar ridge, with need for maxillary sinus floor augmentation. The patients also committed to returning for follow-up appointments and to maintaining adequate oral hygiene. Patients were excluded if they had a history of neoplastic disease treated with radiotherapy or chemotherapy, if they were pregnant or breastfeeding, if they were receiving treatment or were affected by an illness that could have an effect on bone homeostasis, allergy to any of the materials used, and sinus pathologies, or if they were smokers.

Cone-beam computed tomography scans of the posterior maxillary region were obtained to measure the height of the posterior maxillary bone and the size of the maxillary sinus. The CT scans were also used to evaluate possible sinus pathologies (Figure 1).

Eight patients with a mean age of $55.4 \pm 9.2$ years were included in this study. They comprised sixteen atrophic maxillary sinuses to be grafted prior to implant placement. The patients were randomly divided using online-based software available at http://www.randomization.com/ into two groups according to the material used, control group (CG) $(n=8)$ with Bio-Oss only and test group (TG) $(n=8)$ with BioOss combined with bone marrow concentrate obtained by the BMAC method, and each patient had the same graft material placed in each sinus. Following the principles of the guided bone regeneration technique, collagen membranes were placed over the bone window for all sinus floor augmentation procedures in both groups.

All patients were dentally rehabilitated using osseointegrated implants and fixed prostheses at the end of the study.

2.1. BMAC Method. According to the instructions by the manufacturer, bone marrow was harvested and processed directly in the operating room using the BMAC system (Bone 


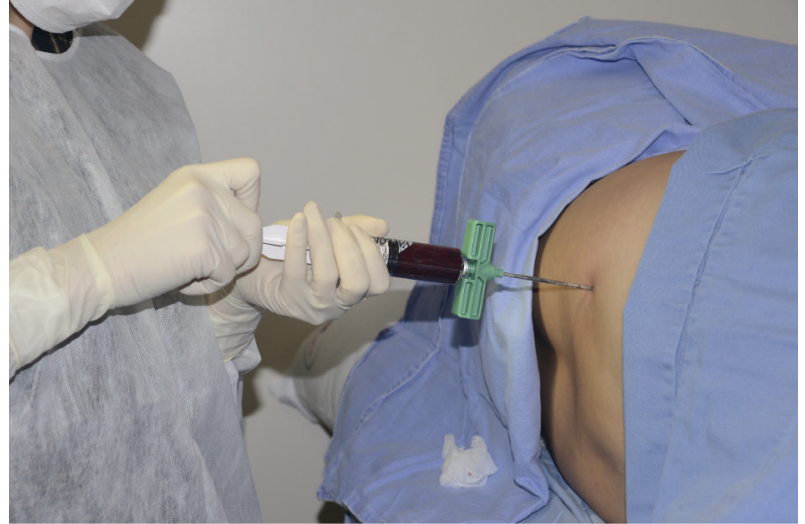

Figure 2: Bone marrow was collected from all the patients by aspiration through a puncture $2 \mathrm{~cm}$ laterocaudally from the superior posterior iliac crest.

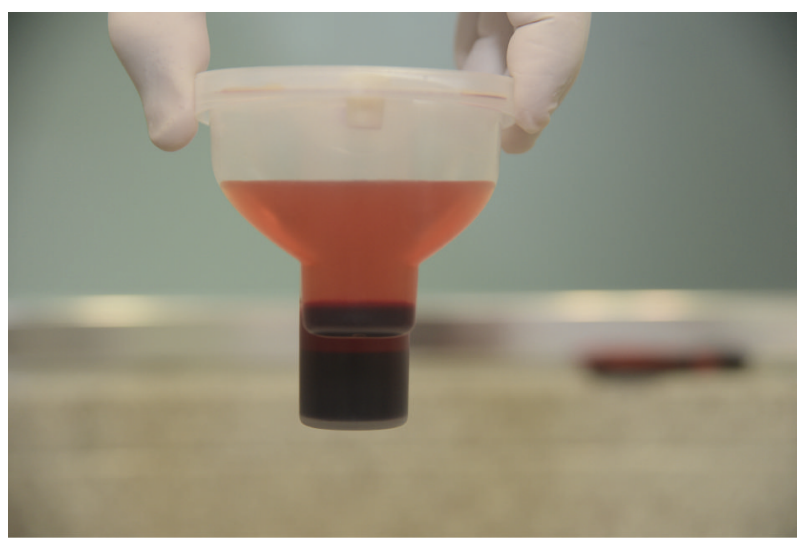

FIgURE 3: After centrifugation for 14 minutes, two phases were obtained within the container: (1) the supernatant plasma above and (2) precipitated bone marrow cells concentrate below.

Marrow Procedure Pack, Harvest Technologies, Plymouth, MA, USA). Briefly, in an outpatient setting and using local anesthesia (2\% xylocaine without a vasoconstrictor), $30 \mathrm{~mL}$ of bone marrow was collected from all the patients by aspiration through a puncture $2 \mathrm{~cm}$ laterocaudally from the superior posterior iliac crest, using a bone marrow needle (included in the pack), with $30 \mathrm{~mL}$ syringes previously heparinized ( $1 \mathrm{~mL}$ of $5.000 \mathrm{U} / \mathrm{mL}$ heparin) (Figure 2).

The syringe containing $30 \mathrm{~mL}$ of bone marrow was connected to a filter bag, to which $8 \mathrm{~mL}$ of ACD-A anticoagulant was added. After appropriate homogenization, new syringe and needle were connected and the filtered $30 \mathrm{~mL}$ was removed. The bone marrow aspirate was then transferred into specific process disposables, which were placed in a SmartPReP2 centrifuge. After centrifugation for 14 minutes, two phases were obtained within the container, that is, the supernatant plasma and the precipitated bone marrow cells concentrate (Figure 3 ). The plasma was removed using specific syringes provided in the kit and the cell concentrate was resuspended and approximately $4 \mathrm{~mL}$ was aspirated.

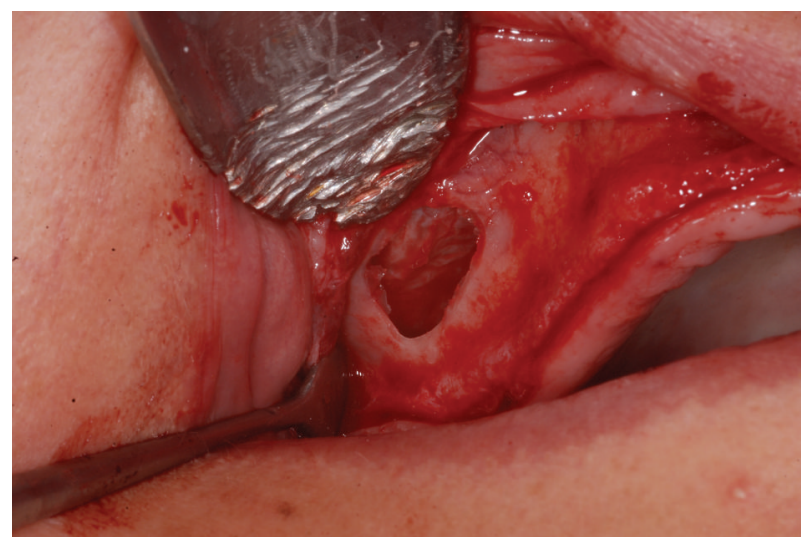

Figure 4: The Schneiderian membrane seen through the lateral window in the maxillary sinus.

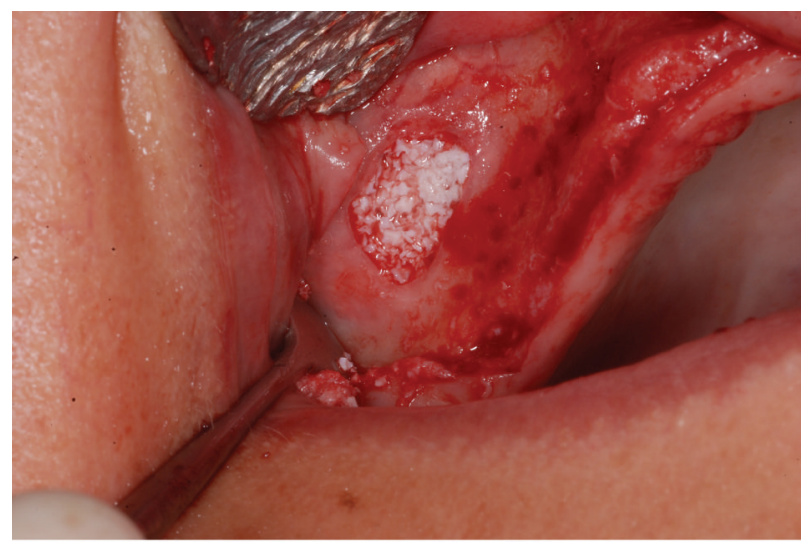

FIGURE 5: Maxillary sinus was grafted with xenogeneic bone from bovine hydroxyapatite.

2.2. Surgical Procedure. A lateral window was prepared using number 3 PM spherical diamond bur (Medical Burs Ind. e Com. de Pontas e Brocas Cirúrgicas Ltda. Cotia, SP, Brazil) on the buccal aspect of the maxillary sinus. The Schneiderian membrane was carefully released (Figure 4) using specific curettes (Neodent, Curitiba, PR, Brazil) and the maxillary sinuses were grafted with xenogeneic bone from bovine hydroxyapatite, (1-2 mm Bio-Oss, Geistlich Biomaterials, Wolhusen, Switzerland) (Figure 5), either alone or combined with the bone marrow concentrate (Figure 6).

A collagen membrane of porcine origin (Bio-Gide, Geistlich Biomaterials, Wolhusen, Switzerland) was used to cover the graft and the osteotomy of the lateral maxillary sinus wall, thus impeding migration of soft tissue to the graft region. After 6 months, 16 bone biopsies (one per sinus) were taken using a trephine bur $(2.0 \mathrm{~mm}$ in diameter and $18 \mathrm{~mm}$ in length) (Figure 7). Immediately after that, the implants (Blackfix, AS Technology, São José dos Campos, Brazil) were placed (Figure 8). The bone biopsies were fixed in $4 \%$ formaldehyde solution (Merck, Darmstadt, Germany). The suture was made with ETHILON-Nylon 4-0 (Ethicon, MA, USA). After the surgery, each patient was prescribed 


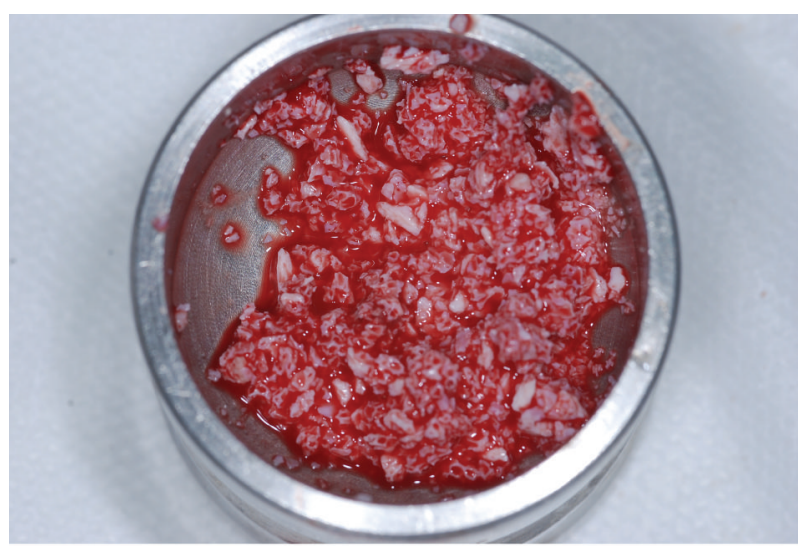

Figure 6: Xenograft combined with the bone marrow concentrate, immediately before being used.

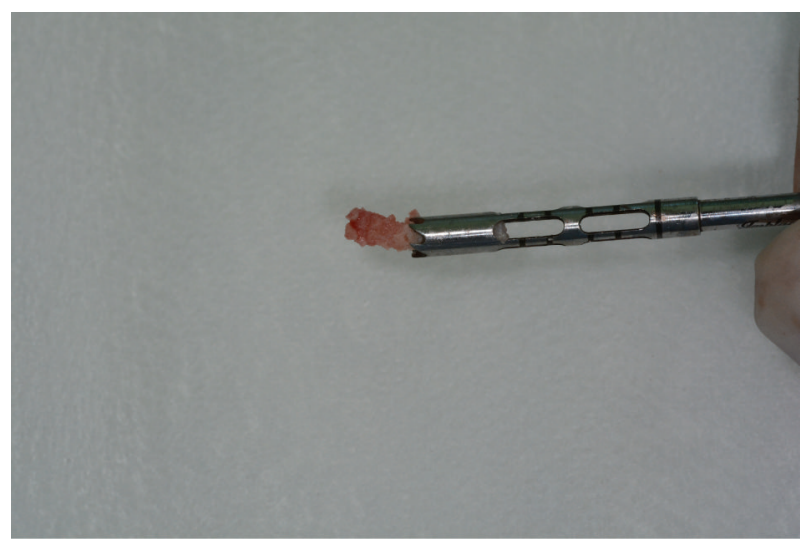

FIGURE 7: Bone specimens removed by a trephine bur.

Amoxicillin $500 \mathrm{mg}$ (12/12 hours for 5 days). All patients were rehabilitated with metal-ceramic prosthesis over the implants six months after its installation.

2.3. Histological Analysis and Histomorphometric Measurements. The histological analysis was performed at the histopathology laboratory of the São Leopoldo Mandic Dental School (Campinas, SP, Brazil). The biopsies underwent decalcification in 10\% EDTA for 36 hours and then processed following a conventional histological method for hard tissue. Subsequently, the samples were embedded in paraffin and 7micrometer sections were cut. The entire area of the trephine biopsy above the native bone of the sinus was defined as region of interest and histomorphometrically evaluated.

The fragments were Masson's trichrome-stained and four different areas of each fragment were evaluated in the histology slides (upper left, lower left, upper right, and lower right), which were then averaged out.

Digital images were captured using a CCD digital camera (RT Cor., diagnostic instruments, Sterling Heights, MI, USA) coupled with an optical microscope (1.25x magnification). The digital images were merged to create a single image for each histological cut, using Adobe Photoshop Elements 2.0

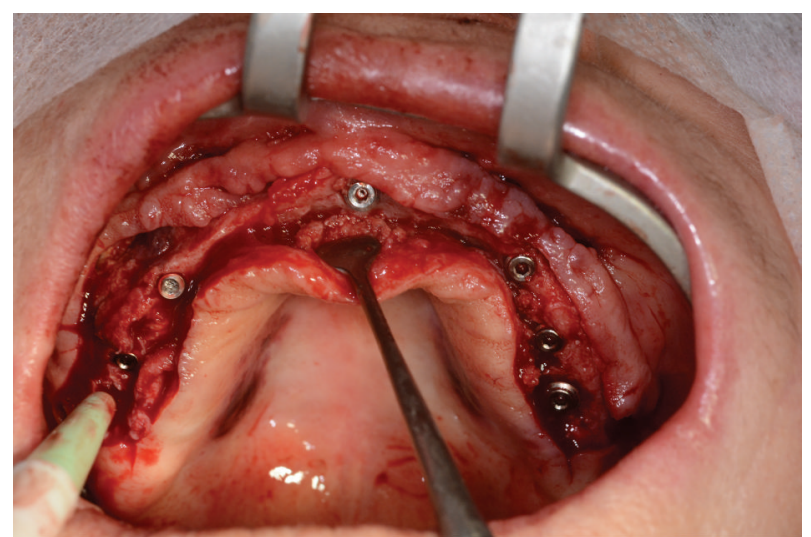

Figure 8: Dental implants (Blackfix, AS Technology, São José dos Campos, Brazil) immediately after they are placed.

software (Adobe Systems, San Jose, CA, USA) (Figures 9(a) and $9(b))$.

Two previously trained examiners (AAP and ACA) blindly examined the images. Whenever disagreement occurred, the specimen was reevaluated and a consensus was reached. The examiners traced new bone formation on all the images using ImageJ Pro Plus 4.5 for Windows software (National Institute of Health, NIH, USA). The following parameters were considered for histomorphometry: (1) nonvital mineralized tissue (NVMT), (2) vital mineralized tissue (VMT), and (3) nonmineralized tissue (NMT). All the results were noted in square micrometers and, subsequently, stated as a percentage of the total area.

2.4. Statistical Analysis. For analysis of the nonvital mineralized tissue (NVMT), vital mineralized tissue (VMT), and nonmineralized tissue (NMT) parameters, values were stated as a percentage of the area evaluated. The nonparametric Mann-Whitney test was applied with correction using the Sidak-Bonferroni test for the statistical analysis. A value of $p<0.05$ was considered significant.

\section{Results}

No membrane perforation was seen during the sinus lift procedures. At least two implants were placed in each previously grafted sinus and all of them were osseointegrated. Loading was applied after a 6-month healing period.

CG and the TG showed percentages of vital mineralized tissue (VMT) area of $27.30 \pm 5.55 \%$ and $55.15 \pm 20.91 \%$, respectively. The same groups showed percentages of nonvital mineralized tissue (NVMT) area (represented by remaining Bio-Oss particles) of $22.79 \pm 9.60 \%$ and $6.32 \pm 12.03 \%$, respectively. Finally, the percentages of nonmineralized tissue $(\mathrm{NMT})$ area were $49.90 \pm 7.64 \%$ and $38.53 \pm 13.08 \%$, respectively (Table 1). 


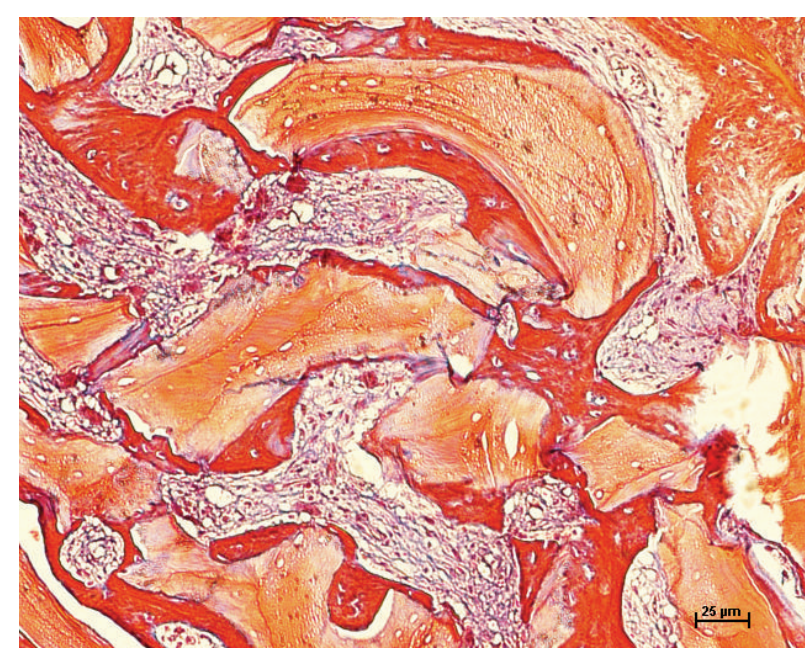

(a)

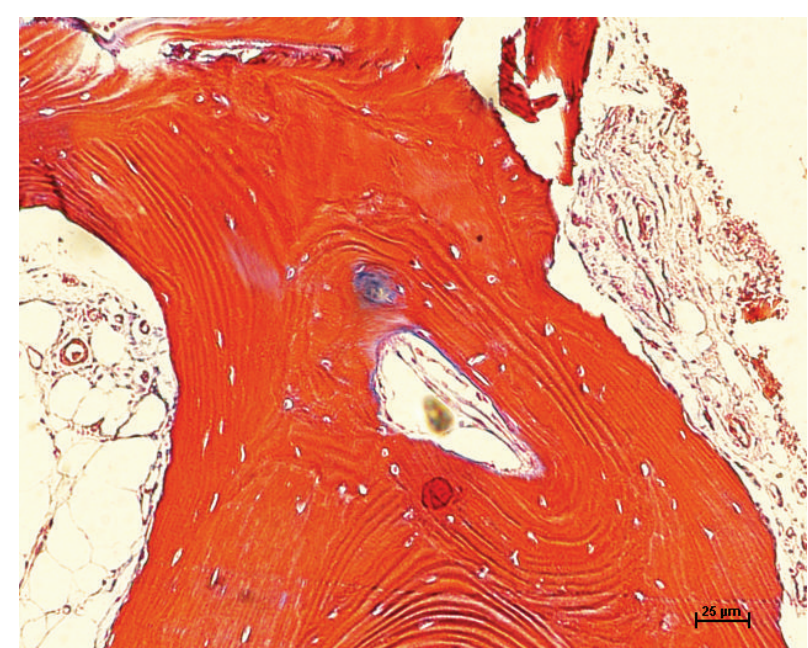

(b)

Figure 9: (a) Histological image of control group, stained by Masson's trichrome technique. Augmentation 20x. (b) Histological image of experimental group, stained by Masson's trichrome technique. Augmentation 20x.

TABLE 1: Statistical comparison of mean values (in percentage, \%) between CG and TG.

\begin{tabular}{lccc}
\hline \multicolumn{4}{c}{ Histomorphometric intergroup analysis } \\
Tissues & CG & TG & $p$ value \\
& $22.79 \pm 9.60$ & $6.32 \pm 12.03$ & 0.006 \\
NVMT & $27.30 \pm 5.55$ & $55.15 \pm 20.91$ & 0.002 \\
VMT & $49.90 \pm 7.64$ & $38.53 \pm 13.08$ & 0.09 \\
NMT &
\end{tabular}

NVMT: nonvital mineralized tissue, VMT: vital mineralized tissue, NMT: nonmineralized tissue, CG: control group, and TG: test group.

Statistically significant $p \leq 0.05$ (Mann-Whitney with correction by SidakBonferroni test).

\section{Discussion}

Surgery to augment the maxillary sinus is a well-documented method to generate adequate amount of bone for implant installation in the posterior maxilla [21-25], for which various types of bone grafts have been tested.

The autologous bone graft technique is considered the gold standard because of its osteoinductive, osteoconductive, and osteogenic characteristics. Nevertheless, it presents some drawbacks, especially regarding operative morbidity, namely, the need for two or more surgical sites in cases of greater amount of donor tissue, including extraoral sources. This raises the operative risk and surgical costs and generates postoperative discomfort, causing fewer patients to opt for this approach [4]. Consequently, a search for bone biomaterials that could replace the autologous bone has taken place. Nonetheless, these are not always graced with the advantages of osteogenesis and osteoinduction inherent of the autologous grafts [3].

Biomaterials that feature the physical, chemical, and mechanical characteristics of autologous bone have become increasingly desired, given the need for further use of the grafted area for the installation of dental implants. The literature reports on a xenogeneic bovine bone substitute, Bio-Oss, as a biomaterial with very similar characteristics to those of human bone, including osteoconduction [26-29]. Lyophilized xenogeneic bone tissue, or other bone substitute graft materials, lacks factors that promote osteogenesis and osteoinduction. In turn, this increases healing time compared to autologous bone, reaching a waiting period for implant placement ranging from six to eight months. This is greater than the time required for autologous grafts, which feature live cells and growth factors, therefore fulfilling their osteogenic and osteoinductive potentials [3]. Such properties reflect positively on the time required for bone healing, which may vary from four to six months [30].

Based on the aforementioned properties, methods to enhance bone substitutes combined with bone marrow cells have been investigated. Studies have described techniques for harvesting and applying fresh bone marrow [31-33] and isolating and expanding mesenchymal stem cells from the bone marrow [33-35] as well as concentrating the bone marrow cells $[18,33,36,37]$ in combination with a mineralized carrier. Despite these various methods, however, there is no consensus on the best alternative for bone remodeling in humans. Regarding the use of cell culture, mesenchymal stem cells must be carefully considered, because they usually require a waiting period of several weeks between harvesting, culture, and transplantation, thus risking contamination [38]. Therefore, in this study, preference was given to test a clinically plausible cell concentration method from a bone marrow aspirate concentrated by centrifugation within a fully closed system. The main reason for this choice was the versatility of the technique and the unlikelihood of contamination due to the fact that the system is closed. As stated by Sakai et al. [36], "to standardize the bone marrow transplantation for bone regeneration, a simple, safe, clean and cost-effective system is needed." 
In the present study, the waiting period of six months between grafting and reopening for implant placement was adopted, as it stands within the waiting period for autologous (4-6 months) and xenogeneic grafting (6-8 months). However, on the grounds that bone neoformation levels (vital mineralized tissue) in the test group were significantly higher ( $p=0.002)$ than in the control group, $55.15 \pm 20.91 \%$ and $27.30 \pm 5.55 \%$, respectively, one can speculate the possibility of early reopening when using the BMAC method. Sauerbier et al. (2010) achieved $19.9 \%$ of new bone after lifting maxillary sinuses with Bio-Oss associated with the BMAC method. The discrepancy between the results from both studies could probably be justified on the time delay between grafting and reopening surgery. In the study by Sauerbier et al. [37], a healing period of approximately 4.1 months was chosen, but in some cases, reopening occurred after just 3 months. The lack of time standardization for reopening combined with a precocious second intervention may have resulted in lower levels of bone tissue as stated by Sauerbier et al. [37] when compared to the present study, which standardized the reopening procedures at 6 months. Rickert et al. [18] compared the combination of Bio-Oss/BMAC method with Bio-Oss (70\%) associated with autologous bone $(30 \%)$ and detected no significant difference between the groups, further suggesting that the BMAC method improved the reconstructive standard. Nevertheless, the authors also opted for an early reopening time of approximately 3 months after grafting, which also hindered further comparisons between their data and those of the present study.

Regarding the levels of residual particles of Bio-Oss (NVMT), a significantly lower percentage $(p=0.006)$ was observed in the test group compared to the control, $6.32 \pm 12.03 \%$ and $22.79 \pm 9.60 \%$, respectively. This can be hypothesized as an acceleration of the healing process in the test group, which corroborates the VMT results, since a higher rate of bone formation should translate into a higher biomaterial resorption. Regarding the levels of NMT, despite the lack of significant difference between the groups ( $p=$ 0.09 ), a decreasing trend in the amount of nonmineralized tissue in the test group was observed, which was, on average, $10 \%$ lower than in the control group.

There have been very few reports on the use of the biomaterials and techniques investigated in the present preliminary study. Therefore, further investigation is required to substantiate these results.

\section{Conclusion}

This study indicates that the clinical use of bone marrow aspirate concentrate obtained by the BMAC method associated with a xenograft for maxillary sinus elevation resulted in more adequate bone repair than the xenograft alone.

\section{Conflict of Interests}

The authors declare that there is no conflict of interests regarding the publication of this paper.

\section{Authors' Contribution}

Paulo José Pasquali performed data analysis/interpretation and concept/design. Marcelo Lucchesi Teixeira performed drafting paper. Thiago Altro de Oliveira helped in data collection. Luis Guilherme Scavone de Macedo helped in data collection. Antonio Carlos Aloise approved the paper and performed concept/design. André Antonio Pelegrine helped in critical revision of the paper and concept/design.

\section{Acknowledgment}

The authors wish to thank Ms Adriana Zardo João, hematologist, for harvesting the bone marrow used for the study.

\section{References}

[1] G. Chaushu, O. Mardinger, M. Peleg, O. Ghelfan, and J. Nissan, "Analysis of complications following augmentation with cancellous block allografts," Journal of Periodontology, vol. 81, no. 12, pp. 1759-1764, 2010.

[2] P. J. Boyne and R. A. James, "Grafting of the maxillary sinus floor with autogenous marrow and bone," Journal of Oral Surgery, vol. 38, no. 8, pp. 613-616, 1980.

[3] D. J. Prolo and J. J. Rodrigo, "Contemporary bone graft physiology and surgery," Clinical Orthopaedics and Related Research, vol. 200, pp. 322-342, 1985.

[4] S. S. Jensen and H. Terheyden, "Bone augmentation procedures in localized defects in the alveolar ridge: clinical results with different bone grafts and bone-substitute materials," The International Journal of Oral \& Maxillofacial Implants, vol. 24, pp. 218-236, 2009.

[5] C. E. A. Ferreira, A. B. Novaes Jr., V. I. Haraszthy, M. Bittencourt, C. B. Martinelli, and S. M. Luczyszyn, "A clinical study of 406 sinus augmentations with $100 \%$ anorganic bovine bone," Journal of Periodontology, vol. 80, no. 12, pp. 1920-1927, 2009.

[6] T. Jensen, S. Schou, A. Stavropoulos, H. Terheyden, and P. Holmstrup, "Maxillary sinus floor augmentation with Bio-Oss or Bio-Oss mixed with autogenous bone as graft in animals: a systematic review," International Journal of Oral and Maxillofacial Surgery, vol. 41, no. 1, pp. 114-120, 2012.

[7] C. Stacchi, G. Orsini, D. Di Iorio, L. Breschi, and R. Di Lenarda, "Clinical, histologic, and histomorphometric analyses of regenerated bone in maxillary sinus augmentation using fresh frozen human bone allografts," Journal of Periodontology, vol. 79, no. 9, pp. 1789-1796, 2008.

[8] C. M. M. Contar, J. R. Sarot, M. B. da Costa et al., "Fresh-frozen bone allografts in maxillary ridge augmentation: histologic analysis," The Journal of Oral Implantology, vol. 37, no. 2, pp. 223-231, 2011.

[9] L. Cordaro, D. D. Bosshardt, P. Palattella, W. Rao, G. Serino, and M. Chiapasco, "Maxillary sinus grafting with Bio-Oss or Straumann Bone Ceramic: histomorphometric results from a randomized controlled multicenter clinical trial," Clinical Oral Implants Research, vol. 19, no. 8, pp. 796-803, 2008.

[10] J. W. F. H. Frenken, W. F. Bouwman, N. Bravenboer, S. A. Zijderveld, E. A. J. M. Schulten, and C. M. Ten Bruggenkate, "The use of Straumann Bone Ceramic in a maxillary sinus floor elevation procedure: a clinical, radiological, histological and histomorphometric evaluation with a 6-month healing period," Clinical Oral Implants Research, vol. 21, no. 2, pp. 201-208, 2010. 
[11] O. Gurevitch, B. G. S. Kurkalli, T. Prigozhina, J. Kasir, A. Gaft, and S. Slavin, "Reconstruction of cartilage, bone, and hematopoietic microenvironment with demineralized bone matrix and bone marrow cells," Stem Cells, vol. 21, no. 5, pp. 588597, 2003.

[12] S. Kale and M. W. Long, "Osteopoiesis: the early development of bone cells," Critical Reviews in Eukaryotic Gene Expression, vol. 10, no. 3-4, pp. 259-271, 2000.

[13] N. Yamamoto, K. Furuya, and K. Hanada, "Progressive development of the osteoblast phenotype during differentiation of osteoprogenitor cells derived from fetal rat calvaria: model for in vitro bone formation," Biological and Pharmaceutical Bulletin, vol. 25, no. 4, pp. 509-515, 2002.

[14] D. Kaigler, P. H. Krebsbach, P. J. Polverini, and D. J. Mooney, "Role of vascular endothelial growth factor in bone marrow stromal cell modulation of endothelial cells," Tissue Engineering, vol. 9, no. 1, pp. 95-103, 2003.

[15] E. Lucarelli, M. Fini, A. Beccheroni et al., "Stromal stem cells and platelet-rich plasma improve bone allograft integration," Clinical Orthopaedics and Related Research, no. 435, pp. 62-68, 2005.

[16] S. Sauerbier, A. Stricker, J. Kuschnierz et al., "In vivo comparison of hard tissue regeneration with human mesenchymal stem cells processed with either the ficoll method or the BMAC method," Tissue Engineering_Part C: Methods, vol. 16, no. 2, pp. 215-223, 2010.

[17] S. Sauerbier, D. Rickert, R. Gutwald et al., "Bone marrow concentrate and bovine bone mineral for sinus floor augmentation: a controlled, randomized, single-blinded clinical and histological trial-per-protocol analysis," Tissue EngineeringPart: A, vol. 17, no. 17-18, pp. 2187-2197, 2011.

[18] D. Rickert, S. Sauerbier, H. Nagursky, D. Menne, A. Vissink, and G. M. Raghoebar, "Maxillary sinus floor elevation with bovine bone mineral combined with either autogenous bone or autogenous stem cells: a prospective randomized clinical trial," Clinical Oral Implants Research, vol. 22, no. 3, pp. 251-258, 2011.

[19] D. Z. Lee, S. T. Chen, and I. B. Darby, "Maxillary sinus floor elevation and grafting with deproteinized bovine bone mineral: a clinical and histomorphometric study," Clinical Oral Implants Research, vol. 23, no. 8, pp. 918-924, 2012.

[20] S. Sampson, A. Botto-van Bemden, and D. Aufiero, "Autologous bone marrow concentrate: review and application of a novel intra-articular orthobiologic for cartilage disease," The Physician and Sportsmedicine, vol. 41, no. 3, pp. 7-18, 2013.

[21] S. S. Wallace and S. J. Froum, "Effect of maxillary sinus augmentation on the survival of endosseous dental implants. A systematic review," Annals of Periodontology, vol. 8, no. 1, pp. 328-343, 2003.

[22] M. Del Fabbro, T. Testori, L. Francetti, and R. Weinstein, "Systematic review of survival rates for implants placed in the grafted maxillary sinus," International Journal of Periodontics and Restorative Dentistry, vol. 24, no. 6, pp. 565-577, 2004.

[23] E. Nkenke and F. Stelzle, "Clinical outcomes of sinus floor augmentation for implant placement using autogenous bone or bone substitutes: a systematic review," Clinical Oral Implants Research, vol. 20, no. 4, pp. 124-133, 2009.

[24] R. J. Klijn, G. J. Meijer, E. M. Bronkhorst, and J. A. Jansen, “A meta-analysis of histomorphometric results and graft healing time of various biomaterials compared to autologous bone used as sinus floor augmentation material in humans," Tissue Engineering Part B: Reviews, vol. 16, no. 5, pp. 493-507, 2010.

[25] R. J. Klijn, G. J. Meijer, E. M. Bronkhorst, and J. A. Jansen, "Sinus floor augmentation surgery using autologous bone grafts from various donor sites: a meta-analysis of the total bone volume," Tissue Engineering Part B: Reviews, vol. 16, no. 3, pp. 295-303, 2010.

[26] T. Berglundh and J. Lindhe, "Healing around implants placed in bone defects treated with Bio-Oss: an experimental study in the dog," Clinical Oral Implants Research, vol. 8, no. 2, pp. 117-124, 1997.

[27] M. Piattelli, G. A. Favero, A. Scarano, G. Orsini, and A. Piattelli, "Bone reactions to anorganic bovine bone (Bio-Oss) used in sinus augmentation procedures: a histologic long-term report of 20 cases in humans," International Journal of Oral and Maxillofacial Implants, vol. 14, no. 6, pp. 835-840, 1999.

[28] M. Hallman, S. Lundgren, and L. Sennerby, "Histologic analysis of clinical biopsies taken 6 months and 3 years after maxillary sinus floor augmentation with $80 \%$ bovine hydroxyapatite and $20 \%$ autogenous bone mixed with fibrin glue," Clinical Implant Dentistry and Related Research, vol. 3, no. 2, pp. 87-96, 2001.

[29] V. Sollazzo, A. Palmieri, L. Scapoli et al., "Bio-Oss acts on Stem cells derived from Peripheral Blood," Oman Medical Journal, vol. 25, no. 1, pp. 26-31, 2010.

[30] N. Esfahanizadeh, A. R. Rokn, M. Paknejad, P. Motahari, H. Daneshparvar, and A. R. Shamshiri, "Comparison of lateral window and osteotome techniques in sinus augmentation: histological and histomorphometric evaluation," Journal of Dentistry, vol. 9, no. 3, pp. 237-246, 2012.

[31] A. A. Pelegrine, C. E. S. da Costa, M. E. P. Correa, and J. F. C. Marques, "Clinical and histomorphometric evaluation of extraction sockets treated with an autologous bone marrow graft," Clinical Oral Implants Research, vol. 21, no. 5, pp. 535542, 2010.

[32] C. E. S. da Costa, A. A. Pelegrine, D. J. Fagundes, M. de Jesus Simoes, and M. O. Taha, "Use of corticocancellous allogeneic bone blocks impregnated with bone marrow aspirate: a clinical, tomographic, and histomorphometric study," General Dentistry, vol. 59, no. 5, pp. e200-e205, 2011.

[33] A. A. Pelegrine, A. C. Aloise, A. Zimmermann, R. de Mello e Oliveira, and L. M. Ferreira, "Repair of critical-size bone defects using bone marrow stromal cells: a histomorphometric study in rabbit calvaria. Part I: use of fresh bone marrow or bone marrow mononuclear fraction," Clinical Oral Implants Research, vol. 25, no. 5, pp. 567-572, 2014.

[34] M. Nagata, H. Hoshina, M. Li et al., "A clinical study of alveolar bone tissue engineering with cultured autogenous periosteal cells: coordinated activation of bone formation and resorption," Bone, vol. 50, no. 5, pp. 1123-1129, 2012.

[35] F. Chen, X. Feng, W. Wu et al., "Segmental bone tissue engineering by seeding osteoblast precursor cells into titanium mesh-coral composite scaffolds," International Journal of Oral and Maxillofacial Surgery, vol. 36, no. 9, pp. 822-827, 2007.

[36] S. Sakai, H. Mishima, T. Ishii et al., "Concentration of bone marrow aspirate for osteogenic repair using simple centrifugal methods," Acta Orthopaedica, vol. 79, no. 3, pp. 445-448, 2008.

[37] S. Sauerbier, A. Stricker, J. Kuschnierz et al., "In vivo comparison of hard tissue regeneration with human mesenchymal stem cells processed with either the FICOLL method or the BMAC method," Tissue Engineering Part C: Methods, vol. 16, no. 2, pp. 215-223, 2010.

[38] E. Lucarelli, D. Donati, A. Cenacchi, and P. M. Fornasari, "Bone reconstruction of large defects using bone marrow derived autologous stem cells," Transfusion and Apheresis Science, vol. 30, no. 2, pp. 169-174, 2004. 

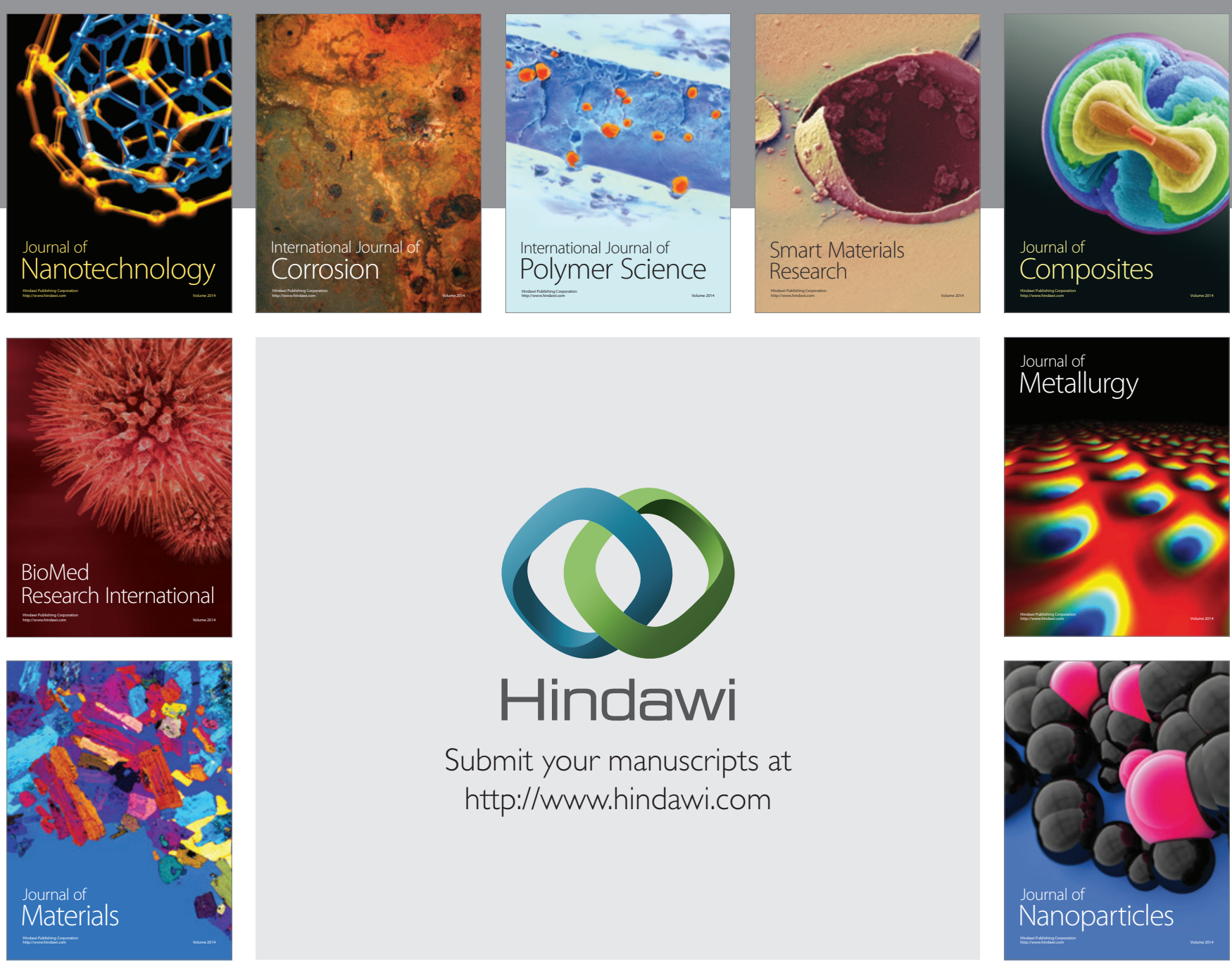

Submit your manuscripts at http://www.hindawi.com
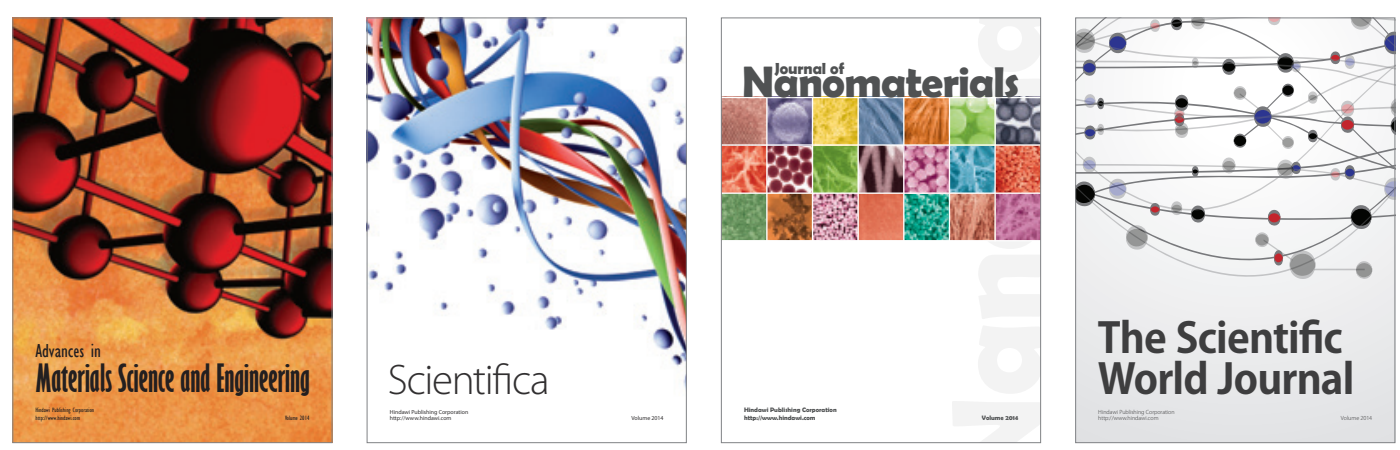

\section{The Scientific World Journal}
